\title{
Análise da assistência à mãe e o cuidado do enfermeiro na Atenção Básica em Saúde para a criança que apresenta microcefalia
}

\author{
Analysis of maternal care and nurse care in Primary Health Care for a child with microcephaly \\ Análisis de la atención materna y de enfermería en la Atención Primaria de Salud de un niño con \\ microcefalia
}

Recebido: 01/07/2022 | Revisado: 01/12/2022 | Aceito: 01/18/2022 | Publicado: 01/22/2022

\author{
Lara Magnabosco Reis Mateus \\ ORCID: https://orcid.org/0000-0003-0555-2026 \\ Universidade Federal do Triângulo Mineiro, Brazil \\ E-mail: laramagnabosco@gmail.com \\ Marina Pereira Rezende \\ ORCID: https://orcid.org/0000-0003-4054-8911 \\ Universidade Federal do Triângulo Mineiro, Brazil \\ E-mail:marina.rezende@uftm.edu.br \\ Ana Rita Marinho Machado \\ ORCID: https://orcid.org/0000-0002-5751-2563 \\ Universidade Federal do Triângulo Mineiro, Brazil \\ E-mail: ana.machado@uftm.edu.br
}

\begin{abstract}
Resumo
O objetivo desta pesquisa foi verificar como se dá a relação/vínculo enfermeiro e mãe de crianças com microcefalia, além de conhecer a assistência de enfermagem no cuidado prestado ao binômio mãe-filho com alterações congênitas, identificando as ações de saúde desenvolvidas à criança com microcefalia. Trata-se de estudo descritivo, com abordagem qualitativa. O estudo foi realizado com enfermeiros das Estratégias de Saúde da Família (ESF) de um Município do Triângulo Mineiro. Para a coleta de dados foi utilizada uma entrevista semiestruturada e o material final foi submetido a Análise de Conteúdo. Como resultado obtivemos que a análise da assistência gera pontos dificultadores como a falta de especialização que auxilia no aprimoramento dos profissionais e os pontos facilitadores estão no processo de discernimento da família em entender o cuidado/assistência a ser prestado, para isso as barreiras dificultadoras devem ser quebradas. Concluiu-se com o presente estudo trouxe reflexões importantes acerca das ações, do conhecimento e diversos pontos facilitadores/dificultadores sobre a análise de assistência as mães de crianças que apresentam microcefalia e como os enfermeiros lidam com a necessidade de promover a adesão das famílias.
\end{abstract}

Palavras-chave: Enfermeiro; Microcefalia; Assistência de enfermagem; Estratégia Saúde da Família.

\begin{abstract}
The objective of this research was to verify how the nurse-mother relationship/bond of children with microcephaly occurs, in addition to knowing the nursing care in the care provided to the mother-child binomial with congenital alterations, identifying the health actions developed for the child with microcephaly. This is a descriptive study with a qualitative approach. The study was carried out with nurses from the Family Health Strategies (FHS) of a municipality in the Triângulo Mineiro region. For data collection a semi-structured interview was used and the final material was submitted to Content Analysis. As a result, we found that the analysis of care generates complicating points such as the lack of specialization that helps in the improvement of professionals and the facilitating points are in the family's discernment process to understand the care/assistance to be provided. broken. The present study concluded with important reflections about the actions, knowledge and various facilitating/complicating points on the analysis of care for mothers of children with microcephaly and how nurses deal with the need to promote family adherence.
\end{abstract}

Keywords: Nurse; Microcephaly; Nursing care; Family Health Strategy.

\section{Resumen}

El objetivo de esta investigación fue verificar cómo se da la relación / vínculo enfermera-madre de los niños con microcefalia, además de conocer el cuidado de enfermería en el cuidado brindado al binomio madre-hijo con alteraciones congénitas, identificando las acciones de salud desarrolladas para el niño con microcefalia. Se trata de un estudio descriptivo con enfoque cualitativo. El estudio se realizó con enfermeras de las Estrategias de Salud de la Familia (ESF) en un municipio de la región Triângulo Mineiro. Para la recolección de datos se utilizó una entrevista semiestructurada y el material final se envió a Análisis de Contenido. Como resultado, encontramos que el análisis del cuidado genera puntos de complicación como la falta de especialización que ayuda en la superación de los 
profesionales y los puntos facilitadores están en el proceso de discernimiento de la familia para comprender el cuidado / asistencia a brindar. El presente estudio concluyó con importantes reflexiones sobre las acciones, conocimientos y diversos puntos facilitadores / complicadores sobre el análisis de la atención a las madres de niños con microcefalia y cómo las enfermeras abordan la necesidad de promover la adherencia familiar.

Palabras clave: Enfermera; Microcefalia; Atención de enfermería; Estrategia de Salud de la Familia.

\section{Introdução}

Foi na Conferência Internacional sobre cuidados primários à saúde, realizada em 1978, em Alma-Ata que se discutiu pela primeira vez a definição sobre a Atenção Primária à Saúde (APS) reafirmando a saúde como um direito universal, além de, representar o primeiro nível de contato com a comunidade, onde passaram a serem cuidados de forma holística nos lugares que vivem e trabalham, sendo um processo contínuo de assistência à saúde conforme as necessidades da população (Declaração De Alma-Ata, 1978).

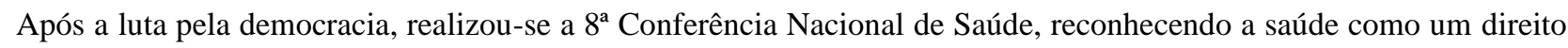
de todo cidadão e dever do Estado, além da recomendação e organização de um Sistema Único de Saúde (SUS). Com a promulgação da Constituição Federal em 1988, foram definidas as diretrizes do SUS em: universalização, equidade, integralidade, descentralização, hierarquização e a participação da comunidade (Informes Técnicos Institucionais USP, 2000).

Em 1991, o Ministério da Saúde implantou o Programa de Agentes Comunitários de Saúde (PACS), onde o governo dá enfoque a família e não apenas ao indivíduo. Esse programa deu início com as experiências advindas de outros estados que utilizavam da cobertura extensa dos serviços de saúde ajudando na diminuição da mortalidade infantil e materna. Diante disso, nos dias 27 e 28 de dezembro de 1993, foi realizada uma reunião convocada pelo ministério da saúde, sobre o tema Saúde da Família, onde surgiu a proposta do Programa de Saúde da Família (PSF), que deveria inserir novos profissionais ao PACS (Viana \& Dal Poz, 2005).

Assim, em 1994, o Ministério da Saúde instituiu a mudança do PSF para Estratégia Saúde da Família (ESF) com intuito de reorganizar a prática de atenção à saúde e melhorar a qualidade de vida da população.

As Unidades Básicas de Saúde (UBS) são os locais onde atuam as ESF, e outras modalidades de equipes de atenção básica como, os Consultórios na Rua, e constituem a mudança do modelo assistencial, a fim de acolher a comunidade e todas as suas necessidades (Brasil, 2012; Leite, 2017).

A Enfermagem é uma das profissões que está presente na UBS, sua regulamentação e atribuições estão presentes no

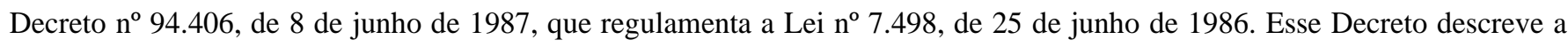
profissão de enfermagem juntamente com suas atribuições, e não faz distinção de seu trabalho nos níveis de atenção à saúde (Brasil, 1987; Soares et al., 2013).

O enfermeiro tem como instrumento primário o acolhimento, e leva ao usuário o atendimento holístico de acordo com a sua necessidade. Para que, esse atendimento aconteça é necessário todo um processo de respeito e confiança entre o usuário e o profissional, levando em conta todo atributo cientifico que o enfermeiro possui (Oliveira et al., 2015). Em relação ao binômio mãe-filho é necessário acolher e acompanhar visando a assistência humanizada e realidade familiar (Rocha, Andrade, 2017).

Segundo o Ministério da Saúde, uma das várias atribuições do enfermeiro, é a consulta de enfermagem visando a promoção da saúde e melhoria da qualidade de vida do binômio mãe-filho. Para o Ministério da Saúde e conforme garantido pela Lei do Exercício Profissional, regulamentada pelo Decreto $n^{\circ}$ 94.406/87, o enfermeiro pode acompanhar inteiramente o pré-natal de baixo risco na rede básica de saúde, demonstrar sua competência técnica e científica realizar escuta qualificada, acolhimento, propiciando assim, a criação de um vínculo, exercendo papel educativo e mudança de maneira positiva na vida dessa gestante (Brasil, 2012).

A infecção que causa a microcefalia apresenta sintomas como, doença febril, acompanhada por cefaleia, exantema, mal-estar, edema e dores articulares. Essa infecção é vinda do vírus da Zika que é um flavivirus da família flaviridae, 
transmitido pelo mosquito Aedes aegypti. Originalmente foi encontrado no ano de 1947 na floresta de Zika (motivo que levou ao nome do vírus), região localizada próximo a Entebbe, na Uganda, sendo isolado na fêmea de um macaco da raça Rhesus febril (Vasconcelos, 2015).

O vírus da Zika pode ser transmitido por via transfusional, perinatal, e existem relatos de transmissão ocupacional em laboratórios e por relação sexual (Reis, 2015).

A microcefalia é a malformação congênita em que o cérebro não desenvolve adequadamente, tornando o perímetro cefálico menor. As crianças que apresentam o perímetro cefálico menor podem ser cognitivamente normais, porém a maioria é acompanhada de alterações motoras e cognitivas, como atraso no desenvolvimento neuropsicomotor e, as funções sensitivas também ficam comprometidas. Esse aumento dos casos de infecção pelo vírus zika requer maior cuidado da gestante durante o acompanhamento pré-natal de baixo-risco, sendo realizado pelo enfermeiro (Brasil, 2016).

As alterações no desenvolvimento neuropsicomotor causadas pelo vírus da Zika em recém-nascidos e crianças devem oferecer estimulação precoce e realização de estratégias de desenvolvimento infanto-juvenil devido as transformações que ocorrem no organismo e principalmente a maturação neurológica, comportamental, sensorial, cognitivo, linguagem e as relações socioafetivas, levando assim a aprendizagem de necessidades humanas básicas e sociais (Garcia, 2018).

Segundo o Ministério da Saúde (2016) e diante do exposto, criou-se um Protocolo devido aos casos de Microcefalia que tiveram uma alteração crescente no ano de 2015 pelo país, tornando a microcefalia um agravo emergencial de saúde pública que está impactando o binômio mãe-filho.

O nascimento de uma criança pode trazer alterações na vida familiar e principalmente na vida da mãe, principalmente quando se tem uma criança com microcefalia, o que torna necessário vigilância e acompanhamento do binômio mãe-filho pelos profissionais de saúde. Assim, cabe ao enfermeiro, fornecer informações a puérpera e sua família no crescimento e desenvolvimento da criança, sanando dúvidas, anseios e acolhendo toda a família (Brasil, 2016).

Diante do exposto este estudo teve como objetivos verificar como se dá a relação/vinculo enfermeiro e mães de crianças com microcefalia e conhecer a assistência de enfermagem no cuidado prestado ao binômio mãe-filho com alterações congênitas, identificando as ações de saúde desenvolvidas à criança com microcefalia.

\section{Material e Métodos}

Tratou-se de estudo descritivo, com abordagem qualitativa. O estudo descritivo contribui para análise e descrição de um determinado acontecimento (Polit et al., 2011) e a abordagem qualitativa procura investigar para compreender a lógica interna de grupos, instituições e atores quanto a sua veracidade (Minayo, 2008).

Além disso, o método qualitativo, que é estabelecido pela empiria e sistematização progressiva de conhecimento, assegura desvelar processos ainda pouco conhecidos inerentes a determinados grupos, propiciando a concepção de novos questionamentos, revisão, indicadores e elaboração de novas hipóteses, conceitos e categorias (Minayo, 2008).

O estudo foi realizado com enfermeiros (as) que trabalham nas Estratégias de Saúde da Família (ESF) do Município de Uberaba.

Em abril de 2009, o prefeito de Uberaba, sancionou a lei de nº 10.739 sobre organização da Saúde da Família no município, além da composição das ESF. O município de Uberaba conta com 3 distritos sanitários (Abadia, Mercês e Boa Vista), sendo que cada um possui cerca de seis áreas de abrangência na zona urbana e de uma a três áreas na zona rural. Uberaba detêm 53 equipes de saúde da família, dentre elas, 48 localizam-se na zona urbana e 5 na zona rural. Das equipes que atuam na cidade, 22 estão presentes no Distrito Sanitário I, 15 no Distrito Sanitário II e 16 no Distrito Sanitário III. As unidades funcionam de segunda a sexta-feira das 7 às 17 horas. 
Foram incluídos no estudo enfermeiros que estiveram desenvolvendo atividades laborais no período da coleta de dados, além de possuírem idade igual ou superior a 18 anos e consentirem participar do estudo. E, foram excluídos os enfermeiros que estiveram afastados do trabalho no período da coleta dos dados ou não forem encontrados após três tentativas para agendamento de entrevista.

Para a coleta de dados foi utilizada uma entrevista semiestruturada com os enfermeiros das Estratégias de Saúde da Família de um município do interior de Minas Gerais. O roteiro de entrevista foi composto por duas partes: a primeira com questões referentes aos dados sócio demográficos e profissionais e a segunda com questões referentes ao trabalho desenvolvido pelos enfermeiros com as mães de crianças com microcefalia, além das próprias crianças. Esse roteiro foi submetido à validação aparente e de conteúdo por três peritos na temática e em metodologia da pesquisa, em seguida, foi aplicado teste piloto para adequação dos itens. Ressalta-se que os participantes do estudo piloto não foram incluídos na população do estudo. Após esse levantamento, foi agendado em comum acordo com os participantes um dia e horário para a realização da entrevista, bem como solicitado um ambiente que assegurasse a privacidade. Foi realizada a abordagem individual com todos os enfermeiros aptos a participarem da pesquisa, por turno e setor de trabalho. Neste momento, os pesquisadores realizaram a leitura do Termo Livre e Esclarecido e, após o aceite em participar da pesquisa, receberam o instrumento e as instruções de como preenchê-lo.

As entrevistas foram gravadas e transcritas na íntegra pela pesquisadora e o material resultante foi submetido à análise de conteúdo, na modalidade temática. Essa técnica de análise consiste em identificar os núcleos de significação presentes nas respostas dos participantes que atendam ao problema de pesquisa (Minayo, 2013).

A análise de conteúdo, na modalidade temática, desenvolve-se sistematicamente em três etapas: pré-análise, exploração do material, tratamento e interpretação dos resultados (Minayo, 2013).

Na pré-análise é feita uma leitura exaustiva do material com o intuito de reconhecer as unidades de significação pertinentes ao objetivo do estudo. Em seguida, na etapa de exploração do material, busca-se agrupar as unidades semelhantes em categorias abrangentes. E por fim, procede-se ao tratamento e interpretação dos resultados, podendo utilizar cálculo de frequência simples e inferência com a literatura científica e contexto pesquisado (Minayo, 2013).

Em um primeiro momento foi solicitada a autorização na Secretaria de Saúde para o desenvolvimento da pesquisa, após autorização do secretário Municipal, o projeto foi submetido à Plataforma Brasil, no Comitê de Ética da Universidade Federal do Triângulo Mineiro a fim de cumprir as exigências da Resolução 466/12 do Conselho Nacional de Saúde que regulamenta as normas para a realização de pesquisas que envolvem seres humanos, estando em acordo à Resolução 466/12 do mesmo conselho, sendo aprovado com CAAE: 92050918.7.0000.5154.

Para participação no estudo, os participantes da pesquisa, seja no teste-piloto ou coleta definitiva dos dados, foram consultados quanto ao interesse e disponibilidade e assinaram o Termo de Consentimento Livre e Esclarecido, sendo esclarecidos de todas as peculiaridades da pesquisa. Cabe enfatizar que foi garantido o caráter sigiloso (identificados por números) e voluntário da participação, bem como o comprometimento de manter este caráter na divulgação dos resultados do trabalho.

\section{Resultados}

Ao total de nove enfermeiros disponíveis que tiveram e/ou possuíam contato com criança apresentando microcefalia em sua ESF, apenas seis participaram do estudo, os demais se negaram ou não foram encontrados nas três tentativas realizadas. A idade dos enfermeiros entrevistados variou entre 29 a 43 anos, e tempo de formação em enfermagem 3 a 12 anos; já como atuante em unidade básica variou de 1 ano e seis meses a 12 anos. Todos os enfermeiros participantes do estudo são do sexo feminino. 
Com o intuito de alcançar o objetivo proposto e por meio da análise de conteúdo pela modalidade temática, os resultados foram estruturados em categorias, são elas: conhecimento e assistência do enfermeiro para oferecer assistência à mãe sobre crianças com microcefalia; percepção quanto aos pontos que dificultam e facilitam essa assistência. Diante de algumas falas das enfermeiras, foi possível observar fatores que intercedem em todo o eixo central: assistência, e para tal é necessário o conhecimento.

\subsection{Conhecimento e assistência do enfermeiro sobre crianças com microcefalia}

“[...] na atenção básica eu acho que está um pouco defasado pela falta de treinamento mesmo, o pouco que a gente sabe é um pouco que a gente lê, pesquisa [...]" Enf 1 .

"Vou ser sincera sou enfermeira meio leiga nessa questão [...] mas a minha percepção como enfermeira sem ter experiência é em avaliar o desenvolvimento da criança, o perímetro cefálico e acompanhar ela direitinho" Enf 2.

"Acho minha percepção muito pouca, eu tenho uma criança na área com microcefalia [...] ai eu faço orientações dos serviços da unidade, a gente meio que faz o serviço de logística, psicólogo, medicação, fralda, é isso." Enf 4.

"Nesse tempo que estou na prefeitura, que é pouco tempo, eu não recebi treinamento, entretanto eles compartilham com a gente...manuais, protocolos do ministério [...]” Enf 6.

\subsection{Percepção quanto aos pontos que dificultam e facilitam a análise à assistência}

"Bom, eu acho que a participação da família é imprescindível no cuidado de uma criança com microcefalia, porque na verdade a gente vai passar as orientações, mas quem vai fazer tudo é a família [...] então eu acho que uma equipe multidisciplinar seria muito bom pra dar uma assistência muito melhor [...]. Enf 1

"Eu acho que falta conhecimento cientifico mesmo [...] a gente não consegue ter um conhecimento tão abrangente de tudo, tem que conhecer um pouquinho de cada, de acordo com a necessidade [...]. O engajamento do profissional em ajudar essa família porque às vezes a gente não tem suporte [...]. Enf 3.

"O fator que dificulta um pouco é como não temos disponibilidade o tempo todo de ir a casa da mãe [...] os fatores que facilitam seria, que quando você tem uma mãe bem orientada e ciente do que acontece, uma mãe disposta a ajudar e cuidar da criança facilita muito". Enf 6.

\section{Discussão}

O processo de trabalho do enfermeiro é voltado para a autonomia e conhecimento do mesmo diante das assertividades do dia-a-dia, porém a assistência desenvolvida por este profissional está relacionada à prática, ao conhecimento e aprendizagem, que conduzem a adequação da assistência para com a família e criança.

A vinda de uma criança com microcefalia altera toda estrutura familiar, onde o modelo de cuidados com uma criança saudável é remodelado diante das novas rotinas e cuidados integrais ao nascimento de uma criança com má formação congênita, os pais mudam seus planos e passam a dedicar exclusivamente à criança, trazendo duvidas e questionamentos perante esses novos obstáculos de cuidados. É essencial reconhecer esses pontos e estar ciente sobre a desestabilização na dinâmica familiar e a separação de papel dada a cada familiar mediante sobrecarga frente a notícia da malformação fetal. (Virgens Menezes et al, 2019).

Percebe-se que, há falta de modalidades e especialização que auxiliem no aprimoramento dos profissionais em questão para que assim consigam planejar uma assistência adequada e individualizada que vai além de orientação, mas motive a família a se tornar ativa no cuidado da criança. No estudo de Santos e Resende (2017), o enfermeiro consegue estabelecer as metas para o desenvolvimento de seu trabalho baseando-se no conhecimento adquirido, realizando ações gerenciais e assistenciais, a fim de sanar dificuldades dos familiares de crianças com microcefalia.

Toda assistência apresenta fatores facilitadores ou dificultadores no ponto de vista do trabalhador, e tendem a estarem presentes no resultado final, para isso é necessário dosar as dificuldades e facilidades e perceber até que ponto isso pode 
atrapalhar ou influenciar o cuidado para com a criança com microcefalia. Os pontos dificultadores nada mais são do que barreiras a serem quebradas com a assistência e conhecimento adequado.

Os fatores relacionados às dificuldades visam os familiares como barreira de assistência do profissional perante o cuidado com a criança. O ponto que facilita a assistência a ser prestada está relacionado com o conhecimento e discernimento da família em entender todo o processo de cuidado a ser gerado com a criança, para isso é necessário que o enfermeiro atue no processo de comunicação e esclarecimento de toda assistência. O mesmo acontece no estudo de Rezende Neta, Silva e Silva (2015) sobre os fatores que dificultam a assistência do profissional, em que ações relacionadas ao autocuidado, a baixa escolaridade e a baixa renda dos pais são importantes barreiras para oferecer as orientações abrangentes a criança. O processo educativo é necessário, porém deve ser de acordo com a verdadeira realidade da população que receberá o conhecimento, sendo priorizado a necessidade de cada família de crianças com microcefalia (Queiroz et al, 2016).

\section{Considerações Finais}

O estudo contribuiu com reflexões importantes acerca das ações, do conhecimento e diversos pontos facilitadores/dificultadores sobre a análise de assistência as mães de crianças que apresentam microcefalia e como os enfermeiros lidam com a necessidade de promover a adesão das famílias. É imprescindível destacar o enfermeiro como profissional atuante e de grande fonte de informação e aproximação com as famílias a fim de promover uma assistência abrangente e individualizada com locus estratégicos para promoção do desenvolvimento da criança.

Nota-se também uma carência de estudos acerca dessa temática. Propõem-se estudos futuros em torno deste tema, no sentido de acompanhar melhores ações e atualizações dos profissionais de saúde a fim de terem o conhecimento como aliado no cuidado da criança com microcefalia.

\section{Referências}

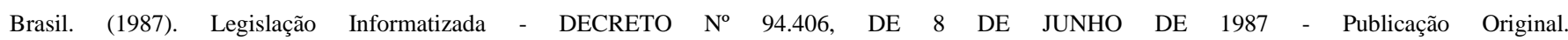
http://www2.camara.leg.br/legin/fed/decret/1980-1987/decreto-94406-8-junho-1987-444430-publicacaooriginal-1-pe.html.

Brasil. Ministério da Saúde. Secretaria de Atenção à Saúde. Departamento de Atenção Básica. (2012). Atenção ao pré-natal de baixo risco / Ministério da Saúde. Secretaria de Atenção à Saúde. Departamento de Atenção Básica. - Brasília: Editora do Ministério da Saúde, p. 318, Série A. Normas e Manuais Técnicos, Cadernos de Atenção Básica, ${ }^{\circ} 32$.

Brasil. Ministério da Saúde. Secretaria de Atenção à Saúde. (2016). Protocolo de atenção à saúde e resposta à ocorrência de microcefalia relacionada à infecção pelo vírus zika [recurso eletrônico] / Ministério da Saúde, Secretaria de Atenção à Saúde. - Brasília: Ministério da Saúde.

Declaração de alma-ata. (2008). In: conferência internacional sobre cuidados primários de saúde, 6-12 set 1978, http://www.opas.org.br/promocao/uploadarq/alma-ata.pdf.

Garcia, L. P. (2018). Instituto de Pesquisa Econômica Aplicada - IPEA. Epidemia do vírus Zika e microcefalia no Brasil: emergência, evolução e Enfrentamento. Texto para discussão. http://www.ipea.gov.br/portal/index.php?option=com_content\&view=article\&id=3249 2:td-2368-epidemia-do-viruszika-e-microcefalia-no-brasil-emergencia-evolucao-eenfrentamento\&catid=411:2018\&directory=1.

Informes técnicos institucionais. (2000). Programa Saúde da Família, Rev Saúde Pública. 34(3), 316-9.

Leite, I. S. (2017). A importância do enfermeiro no programa saúde da família no atendimento ao pré-natal de baixo risco. Caderno Saúde e Desenvolvimento. 10(6). https://www.uninter.com/cadernosuninter/index.php/saude-e-desenvolvimento/article/view/588/447.

Minayo, M. C. S. (2008). O desafio da pesquisa social. In: Minayo, M. C. S. \& Gomes, S. F. D. R. (orgs.). Pesquisa social: teoria, método e criatividade. 27ed. Ed. Vozes.

Minayo, M. C. S. (2013). O desafio do conhecimento: Pesquisa qualitativa em saúde. Ed. Hucitec.

Oliveira, J. R. (2015). Concepções e práticas de acolhimento apresentadas pela enfermagem no contexto da atenção básica à saúde. Rev enferm UFPE on line., Recife, 9(10):1545-1555.

Polit, D. F., \& Beck, C. T. (2011). Delineamento de Pesquisa em Enfermagem. In: Polit DF, Beck CT, Eds., Fundamentos de pesquisa em enfermagem: Avaliação de evidências para prática de enfermagem, Artmed, 247-368.

Queiroz, O., Veraci, M., Brito, M. M. C., Pennafor, L. P. S., \& Bezerra, V. S. M. (2016). Sensibilizando a criança com diabetes para o cuidado de si: Contribuição à prática educativa. Escola Anna Nery Revista de Enfermagem. 20(2):337-343. 
Research, Society and Development, v. 11, n. 2, e11011225489, 2022

(CC BY 4.0) | ISSN 2525-3409 | DOI: http://dx.doi.org/10.33448/rsd-v11i2.25489

Reis, R. P. (2015). Surto de microcefalia no Brasil. Rev Med Minas Gerais. 25(4):463-465. http://rmmg.org/artigo/detalhes/1858.

Rezende-Neta, D. S., Silva, A. R. V., \& Silva, G. R. F. (2015). Adesão das pessoas com diabetes mellitus aos autocuidados com os pés. Revista Brasileira de Enfermagem. 68(1):111-116.

Rocha, A. C., \& Andrade, G. S. (2017). Atenção da equipe de enfermagem durante o pré-natal: percepção das gestantes atendidas na rede básica de Itapuranga - GO em diferentes contextos sociais. Revista Enfermagem Contemporânea. https://www5.bahiana.edu.br/index.php/enfermagem/article/view/1153.

Santos, M. R., \& Resende, F. A. (2017). A importância do trabalho do enfermeiro da atenção básica frente à ameaça epidêmica de arboviroses. Revista Brasileira de Ciências da Vida, 5(2).

Soares, C. E. S., Biagolini, R. E. M., Bertolozzi, M. R. (2013). Atribuições do enfermeiro na unidade básica de saúde: percepções e expectativas dos auxiliares de enfermagem. Rev. \& esc. enferm. USP, 47(4):915-921. http://www.scielo.br/scielo.php?script=sci_arttext\&pid=S0080$62342013000400915 \& \operatorname{lng}=\mathrm{en} \& \mathrm{nrm}=\mathrm{iso}$.

Vasconcelos, P. F. C. (2015). Doença pelo vírus Zika: um novo problema emergente nas Américas. Rev Pan-Amaz Saude, 6(2):9-10.

Viana, A. L. D’. A., \& DalPoz, M. R. (2005). Reforma do Sistema de Saúde no Brasil e o Programa de Saúde da Família. PHYSIS: Rev. Saúde Coletiva, $15: 225-264$.

Virgens-Menezes, M., Neto, J. M., Leal, C., Vasconcelos, A. P., Aragão, H., Silva, N., \& Da-Luz, L. (2019). Dificuldades E Estratégias Da Família No Cuidado Da Criança Portadora De Microcefalia. Rev. Enfermagem Atual In Derme, 88(26):7. 